\title{
Actividad física y ejercicio en tiempos de COVID-19
}

\author{
Physical activity and exercise in COVID-19 times
}

\author{
Andrés Felipe Villaquirán Hurtado ${ }^{1 \times c v L A C}$, Omar Andrés Ramos ${ }^{2}$ CvLAC, Sandra Jimena Jácome ${ }^{3 \text { CvLAC, }}$ \\ María del Mar Meza4 CvLAC
}

Fecha correspondencia:

Recibido: abril 11 de 2020.

Revisado: junio 6 de 2020.

Aceptado: junio 19 de 2020.

Forma de citar:

Villaquirán Hurtado AF, Ramos OA, Jácome SJ, Meza MdelM. Actividad física y ejercicio en tiempos de COVID-19. Rev CES Med. 2020; Especial COVID-19: 51-58.

\section{Open access}

(c) Derecho de autor

Licencia creative commons

Ética de publicaciones

Revisión por pares

Gestión por Open Journal System

DOl: http://dx.doi.org/10.21615/

cesmedicina.34.COVID-19.6

ISSN 0120-8705

e-ISSN 2215-9177

Sobre los autores:

1. Magíster en Intervención Integral en el deportista. Grupo de Investigación Movimiento Corporal Humano y Calidad de Vida, Universidad del Cauca.

Comparte

F日G. $89 \mathbb{R}^{\circ}$ 图

\section{Resumen}

La situación mundial generada por la actual pandemia del COVID-19 ha propuesto diversas estrategias para mitigar el impacto de esta enfermedad. Dentro de las disposiciones establecidas por la OMS se ha implementado el confinamiento y el distanciamiento social, generando un cambio abrupto en los hábitos y rutinas diarias de las personas, impactando la actividad física, el ejercicio y el deporte. Esta medida de restricción aumenta la exposición al sedentarismo, condición que inicia o deteriora una enfermedad crónica no transmisible previa e incrementa el riesgo de mortalidad por la pandemia. El ejercicio debe ser una estrategia a tener en cuenta en tiempo de cuarentena, con el fin de proteger la salud física y mental de las personas, mantener una condición física saludable y un buen rendimiento atlético, cumpliendo así, con los principios y lineamientos metodológicos de la prescripción del ejercicio y el entrenamiento. El objetivo de esta revisión de tema es establecer la importancia de la actividad física y el ejercicio para el cuidado de la salud y el mantenimiento de la condición física en tiempos de confinamiento por COVID-19.

Palabras clave: Ejercicio; Pandemias; Cuarentena; Sistema inmunológico; COVID-19

\begin{abstract}
The global situation generated by the current COVID-19 pandemic has proposed several strategies to mitigate the impact of this disease. Within the provisions established by the WHO, confinement and social distancing have been implemented, generating an abrupt change in people's daily habits and routines, and impacting their physical activity, exercise and sport. This measure of restriction increases exposure to sedentarism, a condition that initiates or deteriorates a previous chronic non-communicable disease and increases the risk of mortality. Exercise should be a strategy to be taken into account during quarantine, in order to protect the physical and mental health of people, maintain a healthy physical condition and a good athletic performance, thus complying with the principles and methodological guidelines of exercise and training prescription. The objective of this review is to establish the importance of physical activity and exercise for health care and fitness maintenance in times of COVID-19 confinement.
\end{abstract}

Keywords: Exercise; Pandemics; Quarantine; Immune system; COVID-19. 
2. Magíster en Salud Pública. Grupo de Investigación Movimiento Corporal Humano y Calidad de Vida. Universidad del Cauca.

\section{Magíster en Ciencias} de la Educación. Grupo de Investigación Movimiento Corporal Humano y Calidad de Vida. Universidad del Cauca.

4. Especialista en Cirugía General. Universidad del Cauca.
El manejo por parte de los organismos de salud en estos tiempos de mitigación han mostrado escasas estrategias, sin una orientación óptima para mantener a las personas lo más activas posibles.

\section{Introducción}

La situación sanitaria mundial es preocupante por la aparición de la nueva enfermedad infecciosa denominada COVID-19, que afecta gravemente a la población (1). La Organización Mundial de la Salud (OMS) ante la rápida propagación y el fácil contagio dispuso de medidas para combatir la diseminación del virus causante de la enfermedad (2). Dentro de las recomendaciones propuestas para prevenir el contagio, dispuso el lavado de las manos frecuentemente, cubrir la boca y nariz al estornudar o toser, mantener el distanciamiento social, evitar tocarse los ojos, la nariz y la boca, y el confinamiento en casa (2).

El objetivo de esta revisión de tema fue establecer la importancia de la actividad física y el ejercicio para el cuidado de la salud y el mantenimiento de la condición física en tiempos de confinamiento por COVID-19, estableciendo pautas y estrategias para realizar una adecuada prescripción del ejercicio durante este tiempo.

Se realizó una búsqueda bibliográfica en las bases de datos Pubmed, Elsevier, Scielo, Springerlink y Google Académico, con las palabras clave: Pandemia, Ejercicio, Virus, Cuarentena, Sistema Inmunológico y COVID-19. Se revisaron los artículos encontrados de diferentes tipos de investigación, sin hacer exclusión por año de publicación. Se hizo un análisis de las fuentes primarias y se realizó la recapitulación de los aspectos más importantes referentes a la actividad física y al ejercicio.

\section{Estado del arte}

El confinamiento es una de las medidas obligatorias que han sido impuestas por los diferentes gobiernos a nivel internacional debido a la facilidad y rapidez de transmisión, sumado al desconocimiento de los aspectos clínicos, virológicos y epidemiológicos sobre el comportamiento del virus $(3,4)$. Si bien, el confinamiento y el distanciamiento social han sido medidas importantes y prioritarias para la salud pública, estas decisiones también han llevado consigo el cierre temporal y parcial de parques, espacios públicos, gimnasios, escuelas deportivas y centros de acondicionamiento físico, que han generado un impacto negativo sobre el estilo de vida de las personas, disminuyendo las posibilidades para realizar actividad física y ejercicio (5).

Las medidas implementadas pueden generar un detrimento del estado de salud de las personas y aumentar la incidencia de enfermedades no trasmisibles $(6,7)$. Al respecto, se han establecido que los principales cuatro factores de riesgo modificables en común que presentan las enfermedades no trasmisibles son el consumo de tabaco o de alcohol, la alimentación no saludable y la inactividad física (6). Sumado a esto, la actual situación también puede causar alteraciones mentales, la presentación de ansiedad, estrés, irritabilidad, depresión, o miedo por la preocupación de las deudas financieras, la falta de información oportuna sobre la enfermedad y el desempleo $(8,9)$.

\section{Inactividad física y desentrenamiento}

Se estima que a nivel mundial uno de cada cuatro adultos es inactivo físicamente (10) y aunque el impacto de la actividad física y el ejercicio han sido ampliamente demostrados para mantener estable una salud física y mental $(3,8,9)$, el manejo por parte de los organismos de salud en estos tiempos de mitigación han mostrado escasas estrategias, sin una orientación óptima para mantener a las personas lo más activas posibles (9). 
Especial COVID-19 2020 - Pág 53

Realizar actividad física al aire libre guardando la distancia con las demás personas y acatando las indicaciones de las autoridades.
La falta de estímulos óptimos por medio del ejercicio debido a la reducción de las actividades físico-deportivas durante la estancia en casa y el distanciamiento social, traen consigo la pérdida parcial o completa de las adaptaciones fisiológicas, mecánicas, anatómicas y cognitivas, lo que se conoce en el entrenamiento deportivo como el principio de reversibilidad. Este principio explica que, ante la ausencia de estimulación por medio del ejercicio, los efectos conseguidos por el entrenamiento se pierden sino hay una continuidad de los procesos (11). Esa pérdida parcial de la actividad física puede generar una disminución de la calidad de vida con mayor riesgo de caídas, reducción de la fuerza muscular y del rendimiento funcional en adultos mayores $(12,13,14)$.

Así mismo, la inactividad física se ha relacionado con cambios negativos en la salud cardiovascular, tales como disfunción y atrofia cardiaca, estrechamiento luminal vascular, aumento de la rigidez arterial, disfunción endotelial y mayor riesgo de mortalidad cardiovascular (15). Por otro lado, con el desentrenamiento se han reportado cambios sobre el rendimiento físico (16), en la composición corporal con aumentos de la grasa corporal y disminución de la masa magra (17), disminución del consumo máximo de oxígeno, incremento de la frecuencia cardiaca y una menor capacidad muscular en términos de control motor producto de una disminución de la coordinación intra e intermuscular (18).

\section{Pautas generales de actividad física y ejercicio}

\section{Actividad física}

La actividad física durante este tiempo de pandemia puede contribuir a mantener o mejorar la tolerancia al ejercicio, el consumo máximo de oxígeno, la capacidad funcional, la salud cardiovascular, la composición corporal, la fuerza muscular, entre otras (19). Para lograr esos beneficios es importante tener en cuenta las indicaciones de un profesional de la salud o del ejercicio, realizar actividad física al aire libre guardando la distancia con las demás personas y acatando las indicaciones de las autoridades, no practicar actividades grupales, evitar la utilización de equipos y elementos ubicados en parques o espacios públicos, desarrollar actividades con una intensidad moderada dentro de casa y combinarlo con ejercicios como subir escaleras, bailar, saltar, utilizar los diferentes espacios de la vivienda y elementos como la escoba, botellas, maletines, cuerdas que se puedan adecuar para hacer diferentes ejercicios. Además, utilizar las redes sociales para publicar las actividades si eso aumenta la motivación; las actividades deben ser divertidas y en familia $(20,21)$.

\section{Ejercicio físico}

Dentro de los procesos de prescripción del ejercicio, se debe prestar un especial cuidado en la interacción de las actividades físico - deportivas y el sistema inmunológico, el cual es conocido como la defensa del organismo contra mecanismos infecciosos (22). Las investigaciones concuerdan que la práctica regular de ejercicio mejora positivamente la respuesta de las funciones inmunes innatas y adaptativas, generando una reducción de la respuesta inflamatoria sistémica y la disminución del riesgo de infección producido por un efecto inmuno-regulador más eficiente $(23,24)$.

Por esto, es necesario mantener un nivel de vida activo con hábitos de ejercicio en casa, que permitan tener un sistema inmunológico fuerte. Sin embargo, el tipo de ejercicio realizado también influye a la hora de proteger o desestabilizar la respuesta inmunitaria: se ha demostrado cómo el ejercicio interválico de alta intensidad (por sus siglas en inglés, HIIT), puede causar una respuesta inflamatoria aguda moderada, con 
Los profesionales de la salud y del ejercicio deben asumir este reto, buscando las alternativas necesarias para garantizar el acceso de las personas al ejercicio a través de prácticas innovadoras como la construcción de canales de comunicación remota entre profesional y usuario, en donde sesiones virtuales en vivo, videos pregrabados, imágenes, audios o planes escritos y comunicaciones telefónicas pueden fortalecer el autocuidado y el apoyo en la prescripción del ejercicio que vaya de la mano de estas nuevas dinámicas sociales que trajo la pandemia. aumento significativos de interleucina IL-6, IL 8, IL 10, factor de necrosis tumoral a y proteína quimiotáctica monocítica 1 (24). Además, el ejercicio intenso puede producir leucocitosis, producto del aumento de neutrófilos, linfocitos TY B, y células NK (natural killer) en el torrente sanguíneo (25).

No obstante, se debe aclarar que estas respuestas son dadas como respuesta aguda ante el ejercicio y que la práctica regular continua, con una adecuada prescripción, mejora y promueve una mejor respuesta de la función inmune (25).

Lo descrito anteriormente, debe generar diversas recomendaciones en cuanto al ejercicio utilizado durante este tiempo de pandemia. Se deben tener en cuenta las metas y objetivos, y valorar si éstos van dirigidos hacia el mantenimiento de la salud o del rendimiento. Adicionalmente, deben considerarse las pautas generales y específicas, teniendo en cuenta las variantes que existen entre población sana activa o inactiva, niños y adolescentes, adultos mayores y deportistas (26), buscando la seguridad y eficiencia de los estímulos prescritos, permitiendo el desarrollo armónico de la fuerza muscular, la capacidad cardiorrespiratoria, la flexibilidad, la coordinación, la agilidad y el equilibrio, mediante sesiones de trabajo que permitan favorecer o mantener la condición física saludable (27).

La alternancia y variedad durante las sesiones de entrenamiento que incluyan diferentes tipos de ejercicios, con una duración entre 30 a 60 minutos por sesión, entre cinco a siete días por semana, teniendo en cuenta las fases de una sesión de ejercicio que corresponden a la fase de calentamiento, la fase central y la de recuperación, así como también el cuidado adecuado de las cargas y los tiempos de recuperación, pueden favorecer el efecto protector del ejercicio sobre la salud física y mental (28). Los profesionales de la salud y del ejercicio deben asumir este reto, buscando las alternativas necesarias para garantizar el acceso de las personas al ejercicio a través de prácticas innovadoras como la construcción de canales de comunicación remota entre profesional y usuario, en donde sesiones virtuales en vivo, videos pregrabados, imágenes, audios o planes escritos y comunicaciones telefónicas pueden fortalecer el autocuidado y el apoyo en la prescripción del ejercicio que vaya de la mano de estas nuevas dinámicas sociales que trajo la pandemia (29). El uso de las tecnologías puede favorecer la implementación de programas específicos y adaptados a las condiciones y preferencias, permitiendo un control y seguimiento de los procesos. Además, el profesional puede monitorizar y adecuar los componentes de la prescripción del ejercicio como la frecuencia, la intensidad, el volumen, el tiempo y tipo de ejercicios de acuerdo a las necesidades y posibilidades de cada individuo $(30,31)$.

\section{Niños y adolescentes}

En cuanto a los niños y adolescentes se requieren acciones urgentes para incrementar los niveles de actividad deportiva. Estos procesos deben ser graduales y progresivos con actividades lúdicas que involucren el juego y la diversión, siendo prudentes con el número de días y sesiones a realizar evitando la aparición de posibles lesiones. Los ejercicios utilizados deben estar orientados a mejorar la capacidad cardiovascular, la fuerza, la coordinación y la salud ósea, realizar actividades deportivas al aire libre evitando compartir implementos deportivos con otros niños y evitar los juegos que involucren contacto corporal, implementar juegos que promuevan la enseñanza de una buena higiene y las medidas de protección para evitar el contagio, realizar una adecuada limpieza y desinfección de los diferentes juegos e implementos deportivos que tengan en casa (31). 
Especial COVID-19 2020 - Pág 55

Antes de pensar en mejorar el rendimiento o la capacidad física en estos tiempos de pandemia, debe pensarse en un ejercicio seguro y eficiente que cuide la salud tanto física como mental, garantizando la adaptación, el progreso o el mantenimiento de la condición física saludable.

\section{Adultos mayores}

El proceso de envejecimiento aumenta la vulnerabilidad ante el COVID-19 generando un gran impacto sobre la salud física y mental de los adultos mayores. Al respecto, el ejercicio físico puede contribuir a preservar la salud física, la actividad cerebral, la capacidad cognitiva y las funciones mentales, mediante la inclusión de ejercicios aeróbicos, de fuerza muscular, ejercicios de fortalecimiento de la musculatura respiratoria, de equilibrio y flexibilidad, acompañados de tareas y estímulos cognitivos (32).

\section{Deportistas}

Por otra parte, los procesos de entrenamiento de los deportistas deben incluir estrategias que mantengan motivado al atleta, brindando un apoyo interdisciplinar, con entrenamientos individualizados que permitan trabajar los eslabones débiles del control motor, la movilidad y la estabilidad, estableciendo un seguimiento y control de las cargas de entrenamiento que favorezcan el bienestar, el rendimiento y reduzcan el riesgo de lesión, educar al deportista para favorecer el entrenamiento invisible, proveer sesiones alternativas que favorezcan el conocimiento táctico y estratégico del juego.

Además de favorecer el retorno seguro a los entrenamientos después de levantar las medidas de prohibición, las cuales deben darse bajo los protocolos que protejan la salud del deportista, con exámenes médicos y físicos previos, con un seguimiento diario de la salud, estableciendo un regreso gradual por fases que incluyan a todos los actores del deporte (deportistas, entrenadores, jueces, personal médico y de entrenamiento), permitiendo el tiempo necesario y suficiente para cumplir con un proceso de entrenamiento que mejore su rendimiento deportivo y que reduzca la incidencia de lesión (15,16,33).

\section{Conclusiones}

La importancia de mantenerse activo durante esta etapa de confinamiento contribuye significativamente en la prevención de contagio del SARS-CoV-2 y el cuidado de la salud. El ejercicio debe procurar ser individual, específico, atendiendo los objetivos y las metas individuales, con una planeación estructurada y el manejo de las cargas apropiadas. Antes de pensar en mejorar el rendimiento o la capacidad física en estos tiempos de pandemia, debe pensarse en un ejercicio seguro y eficiente que cuide la salud tanto física como mental, garantizando la adaptación, el progreso o el mantenimiento de la condición física saludable.

Por último, es importante mencionar que los niños, adultos mayores o individuos con síntomas o enfermedades cardiovasculares o pulmonares crónicas deben buscar asesoría de un profesional de la salud, para una adecuada y segura prescripción del ejercicio y recordar que hacer cualquier tipo de actividad física es mejor que no hacer nada.

Dado que estamos atravesando por una situación especial atípica, de continuo cambio, de nuevos conocimientos, de avances y retrocesos en el manejo de esta enfermedad, esta revisión permite plantear posteriores investigaciones que mejoren la toma de decisiones por parte de los profesionales del ejercicio y de la salud para mitigar las consecuencias de COVID-19.

\section{Declaración de autores}

Los autores declaramos no tener ningún conflicto de intereses. 


\section{Bibliografía}

1. Trilla A. Un mundo, una salud: la epidemia por el nuevo coronavirus COVID-19. Med Clin (Barc). 2020; 154 (5):175-177. Disponible en: https://doi.org/10.1016/j. medcli.2020.02.002

2. Organización Mundial de la Salud. Brote de enfermedad por coronavirus (COVID-19): orientaciones para el público. [Sitio en internet]. Disponible en: URL https://www. who.int/es/emergencies/diseases/novel-coronavirus-2019/advice-for-public

3. Usher K, Bhullar N, Jackson D. Life in the pandemic: Social isolation and mental health. J Clin Nurs. 2020,00:1-2. Disponible en: https://doi.org/10.1111/jocn.15290.

4. Law S, Leung AW, Xu C. Severe acute respiratory síndrome (SARS) and coronavirus disease-2019 (COVID-19): From causes preventions in Hong Kong. Disponible en: https://doi.org/S1201-9712(20)30192-2.

5. Heffernan K, Young S. Exercise as medicine for COVID-19: an ACE in the hole? Med Hypotheses.2020;142: 1098035. Disponible en: https://doi.org/10.1016/j. mehy.2020.109835.

6. Booth FW, Roberts CK, Thyfault JP, Ruegsegger GN, Toedebusch RG. Role of inactivity in chronic diseases: Evolutionary insight and pathophysiological mechanisms. Physiol Rev. 2017; 97 (4): 1351-1402. Disponible en: https://doi. org/10.1152/physrev.00019.2016.

7. Chen P, Mao L, Nassis G, Hammer P, Ainsworth B, Fuzhing L. Coronaviruses disease (COVID-19): The need to mantein regular physical activity while taking precautions. J Sport Health Sci. 2020; 9 (2): 103-104. Disponible en: https://doi. org/10.1016/j.jshs.2020.02.001

8. Zhang S, Wang Y, Rauch A, Feng W. Unprecedented disruption of lives and work: Health, distrees and life satisfaction of working adults in China one month into the COVID-19 outbreak. Psychiatry Res. 2020;288: 112958. Disponible en: https:// www.sciencedirect.com/science/article/pii/S0165178120306521

9. Carter SJ, Baranauskas MN, Fly AD. Considerations for obesity, vitamin D, and physical activity amidst the COVID-19 pandemic. Obesity (Silver Spring). 2020. Disponible en: https://doi.org/10.1002/oby.22838.

10. Haileamlak A. Physical Inactivity: The major risk factor for non-communicable diseases. Ethio J Health Sci. 2019; 29 (1): 810. Disponible en: https://doi. org/10.4314/ejhs.v29i1.1

11. Esain I, Gil SM, Bidaurrazaga- Letona I, Rodriguez- Larrad A. Effect of 3 months of detraining on functional fitness and quality of life in older adults who regularly exercise. Aging Cli Exp Res. 2019; 31 (4): 503-510. Disponible en: https://doi. org/10.1007/s40520-018-0990-1

12. Esain I, Rodriguez- Larrad A, Bidaurrazaga- Letona I, Gil SM. Health- related quality of life, handgrip strength and falls during detraining in elderly habitual exercisers. Health Qual Life Outcomes. 2017;15 (1): 226. Disponible en: https:// doi.org/10.1186/s12955-017-0800-z. 
13. Correa C, Cunha G, Marques N, Oliveira- Reischak A, Pinto R. Effects of strength training, detraining and retraining in muscle strength, hypertrophy and functional tasks in older female adults. Clin Physiol and Functional Imag. 2015. 36 (4): $306-$ 310. Disponible en: https://doi.org/10.1111/cpf.12230.

14. Kalapotharakos VI, Diamantopoulos K, Tokmakidis SP. Effects of resistance training and detraining on muscle strength and funtional performance o folder adults aged 80 to 88 years. Aging Clin Exp Res. 2010; 22 (2): 134-40. Disponible en: https://doi.org/10.1007/bf03324786

15. Pecanha T, Goessler K, Roschel H, Gualano B. Social isolation during the COVID-19 pandemic can increase physical inactivity and the global burden of cardiovascular disease. Am J Physiol Heart Circ Physiol. 2020; 318: H1441-H1446. Disponible en: https://doi.org/10.1152/ajpheart.00268.2020

16. Jukic I, Calleja-González J, Cos F, Cuzzolin F, Olmo J, Terrados N. Strategies and solutions for teams sports athletes in isolation due to COVID-19. Sports (Basel). 2020; 8(4): 56. Disponible en: https://doi.org/10.3390/sports8040056

17. Suarez-Arrones L, Lara- Lopez P, Maldonado R, Torreno N, De Hoyo M, Nakamura $F Y$, et al. The effects of detraining and retraining periods an fat mass and fat-free mass in elite male soccer players. Peer J. 2019; 7: e7466. Disponible en: https:// doi.org/10.7717/peerj.7466.

18. Guerrero- Calderon B. The effect of short-term and long-term coronavirus quarantine on physical performance and injury incidence in high-level soccer. Soccer and Society.2020. Disponible en: https://doi.org/10.1080/14660970.2020.1772240

19. Wilkinson TJ, Shur NF, Smith AC. "Exercise as medicine" in chronic kidney disease. Scan J Med Sci Sports. 2016; 26 (8): 985-8. Disponible en: https://doi. org/10.1111/sms.12714

20. Hammami A, Harrabi B, Mohr M, Krustrup P. Physical activity and coronavirus disease 2019 (COVID-19): specific recommendations for home-based physical training. Managing Sports and Leisure. 2020. Disponible en: https://doi.org/10.1 $\underline{080 / 23750472.2020 .1757494}$

21. Jurak G, Morrison S, Leskoek B, Kovac M, Hadzic V, Vodicar J, et al. Physical activity recommendations during the coronavirus disease-2019 virus outbreak. J Sport Health Sci. 2020. Disponible en: https://doi.org/10.1016/j.shs.2020.05.003

22. Weyh C, Kruger K, Strasser B. Physical activity and diet shape the immune system during aging. Nutrients 2020; 12 (3). Disponible en: https://doi.org/10.3390/ nu12030622.

23. Xie Y, Wang JP. [Advance in effect of aerobic exercise on immune system and autoimmune diseases]. Sheng Li Xue Bao. 71 (5): 769-782. Disponible en: https:// www.ncbi.nlm.nih.gov/pubmed/31646331

24. Zwetsloot K, John C. Lawrence M, Battista R, Shanley A. High-intensity Interval training induces a modest systemic inflammatory response in active, young men. J Inflamm Res. 2014; 7: 9-17. Disponible en: https://doi.org/10.2147/JIR.S54721 
25. Monteiro PA, Campos EZ, de Oliveira FP, Peres FP, Rosa- Neto JC. Pimentel GD, et al. Modulation of inflammatory response arising from high. Intensity intermittent and concurrent strngth training in phisically active males. Cytokine. 2017; 91: 104-109. Disponible en: https://doi.org/10.1016/i.cyto.2016.12.007

26. Colegio Americano del Deporte. Manual ACSM para la valoración y prescripción del ejercicio 8va Edición. Editorial Paidotribo. 2019.

27. Physical exercise as therapy to fight against the mental and physical consequences of COVID-19 quarantine: Special focus in older people. Prog Cardiovasc Dis. 2020. Disponible en: https://doi.org/10.1016/j.pcad.2020.03.009

28. Rodriguez M, Crespo I, Olmedillas H. exercising in times of COVID-19: what do experts recommend doing within four walls?. Rev Esp Cardiol. 2020. Disponible en: $\underline{\text { http://doi.org/10.1016/j.recesp.2020.04.002 }}$

29. Bormann de Souza B, Fernandes E. COVID-19: the importance of new technologies for physical activity as a public health strategy. Cad Saúde Pública. 2020; 36(5): e00054420. Disponible en: http://doi.org/10.1590/0102-311X00054420

30. Subirats B, Subirats G, Soteras I. Prescripción del ejercicio físico: indicaciones, posología y efectos adversos. Med Clin. 2012; 138 (1): 18-24. Disponible en: http://doi.org/10.1016/j.medcli.2010.12.008

31. Chen P, Mao L, Nassis G, Harmer P, Ainsworth B, FuzHong L. Returning Chinese school-aged children and adolescents to physical activity in the wake of COVID-19: actions and precautions. J Sport Health Sci. 2020: 1-3. Disponible en: http://doi.org/10.1016/j.shs.2020.04.003

32. Woods J, Hutchinson N, Powers S, Roberts W, Gomez-Cabrera M, Radak Z, et al. The Covid-19 Pandemic and physical activity, Sports Med Health Sci. 2020. Disponible en: http://doi.org/10.1016/i.smhs.2020.05.006

33. Primorac D, Matisic V, Molnar V, Bahtijarevic Z, Polasek O. Pre-season football preparation in the era of COVID-19: Croatian football association model. JoGH. 2020; 10(1): 010352. Disponible en: http://doi.org/10.7189/jogh.10.010352 